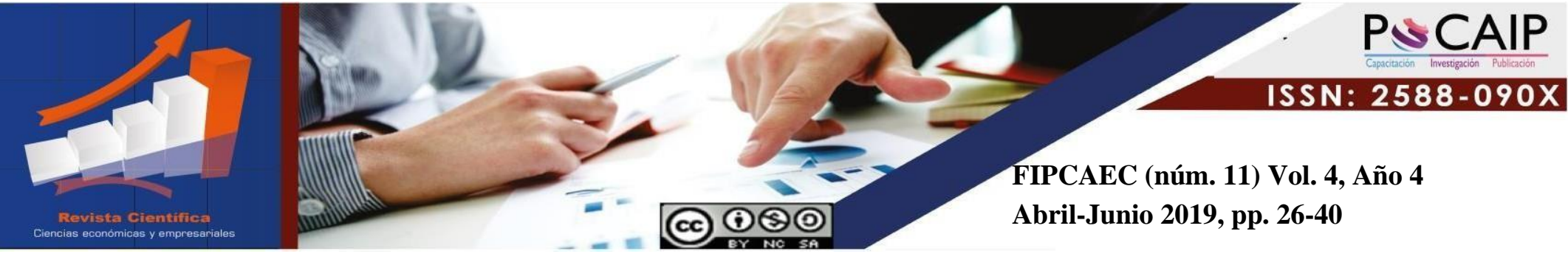

DOI: DOI 10.23857/fipcaec.v4i11.45

\title{
Relaciones interpersonales del gerente educativo en la convivencia escolar
}

\author{
Interpersonal relationships of the educational manager in school life
}

\section{Relações interpessoais do gerente educacional na vida escolar}

\author{
Silvia Patricia Simisterra-Masias ${ }^{1}$ \\ pizafeo@yahoo.es \\ https://orcid.org/0000-0001-8604-5813
}

Correspondencia: pizafeo@yahoo.es

Recepción: 24/ 01/2019 * Aceptación: 19/03/2019 * Publicación: 15/04/ 2019

1 Licenciada en Secretariado Ejecutivo Bilingüe, Área de Nivelación y admisión. Docente de la Universidad Luis Vargas Torres de Esmeraldas, Esmeraldas, Ecuador. 


\section{Resumen}

El presente estudio estuvo dirigido a evaluar las relaciones interpersonales del gerente educativo en la convivencia escolar. El estudio se enmarcó en una investigación de tipo descriptiva con un diseño de investigación no experimental, transeccional y de campo. La muestra estuvo conformada por 115 docentes universitarios que ocupan cargos gerenciales. La recolección de datos se obtuvo a través de un cuestionario estructurado auto administrado contentivo de 25 ítems. La confiabilidad se determinó a través del método de Alfa de Cronbach dando como resultado un rtt de ,985 considerándolo altamente confiable. Se concluyó que existe acuerdo entre los investigados con las relaciones interpersonales basadas en la participación y consenso y un ni acuerdo ni desacuerdo con la resolución de conflictos.

Palabras clave: Relaciones interpersonales; participación; consenso y resolución de conflictos.

\section{Abstract}

This study was aimed at evaluating the interpersonal relationships of the educational manager in school coexistence. The study was framed in descriptive-type research with a non-experimental, transectional and field research design. The exhibition consisted of 115 university professors holding managerial positions. Data collection was obtained through a 25 -item contentious selfmanaged structured questionnaire. Reliability was determined through Cronbach's Alpha method resulting in a $.985 \mathrm{rtt}$ deeming it highly reliable. It was concluded that there is agreement among those investigated with interpersonal relationships based on participation and consensus and a no agreement or disagreement with conflict resolution.

Keywords: Interpersonal relationships; participation, consensus and conflict resolution.

\section{Resumo}

Este estudo teve como objetivo avaliar as relações interpessoais do gerente educacional na vida escolar. O estudo foi enquadrado em uma pesquisa descritiva, com um desenho de pesquisa não experimental, transecional e de campo. A amostra foi composta por 115 professores universitários que ocupam cargos gerenciais. A coleta de dados foi obtida por meio de um 
questionário autoaplicável estruturado, contendo 25 itens. A confiabilidade foi determinada pelo método Alpha de Cronbach, resultando em um rtt de 985, considerando-o altamente confiável. Concluiu-se que há concordância entre os investigados com relações interpessoais com base na participação e consenso e nem concordância ou discordância com a resolução de conflitos.

Keywords: Relações interpessoais; participação; consenso e resolução de conflitos.

\section{Introducción}

Si bien en la realidad, la gestión del gerente educativo como lo ha señalado por Wiemann, M. (2011), se ha caracterizado en algunos casos por relaciones laborales conflictivas, situación que lleva a las relaciones humanas dentro de los espacios educativos a tener un ambiente de trabajo nocivo, donde la resolución de conflictos laborales son realidades cotidianas en el quehacer directivo llevando a generar impases en la relaciones internas del personal que allí laboran. Sin embargo, tal como se ha expuesto Haberman (2008), las relaciones interpersonales del gerente educativo promueven unas relaciones humanas de poca participación, sin propuestas para solucionar ni crear consensos a los conflictos que se presentan en dichas instituciones en pro a las exigencias que su cargo le demanda. En consecuencia, tal vez, poco se propicia la comunicación, empatía y el esfuerzo conjunto entre el personal que labora en los espacios escolares, coartando las probabilidades de motivación, empatía y disposición de empoderamiento que ayuden a ampliar los intereses del personal humano que da vida a la institución. Con base en esta posición, en este artículo se evaluaron las relaciones interpersonales del gerente educativo en la convivencia escolar

\section{Desarrollo}

Dentro de este marco de ideas, Lemus (2003), afirma que una función y una consecuencia de la buena gerencia se constituyen durante el proceso que fluye para que se den las relaciones humanas. Es su propósito, influir en las condiciones de las relaciones interpersonales, pero a la vez influida por ellas. Su función es eminentemente humana, y aun cuando gran parte del éxito de la organización depende de las cualidades personales de los gerentes, también es cierto que las relaciones humanas, además de heredadas biológicas y socialmente, pueden en gran parte ser 
- Se debe mostrar deseos de participación.

- Ser conscientes de los hechos y resultados derivados de las aportaciones individuales de cada miembro del recinto escolar.

- Se debe tener necesaria competencia para hacer dichas aportaciones.

- Debe proporcionarse el tiempo suficiente para que pueda producirse una auténtica participación. El proceso de la participación es lento; es por esto por lo que tiene mayores posibilidades de éxito cuando se aplica a problemas de larga proyección en el tiempo.

- Finalmente, el gerente educativo que promueve la participación debe valorar los puntos de vista y las sugerencias aportadas y llevar a la práctica las decisiones tomadas.

La Resolución de Conflictos: Es lógico suponer que en toda organización durante el tiempo en que los individuos cumplen los procesos de desempeño surgen una serie de conflictos que el gerente debe estar en capacidad de resolver. En efecto, Milkovich y Boudreau (2004), consideran que el conflicto debe dirigirse de manera que se protejan los derechos de los miembros de una organización. Además de los aspectos disciplinarios, pueden surgir conflictos en el trabajo por diversas razones. Algunas organizaciones han diseñado procedimientos que proporcionan un mecanismo para que todos expresen sus desacuerdos.

Estos autores consideran que para ser eficaces, los sistemas de resolución de conflictos deben ser más atractivos que las renuncias o las demandas. Esta norma conduce a cuatro criterios para evaluar dichos sistemas:

- Equidad del acuerdo. Los empleados deben percibir que una organización es capaz y tiene la voluntad de cambiar la situación que provoca el problema.

- Oportunidad del acuerdo. Reducir el período de incertidumbre y la pérdida de beneficios que emanan de la continuación de la disputa, considerando que es una de las principales ventajas de las quejas, en comparación con las demandas.

- Facilidad de utilización. El tiempo y el esfuerzo requerido desde que se entabla hasta que se sigue la apelación debe ser mínimo. 
bien y no quién tiene razón. A medida que el grupo va enfocando lo que está bien, las personalidades e intereses antagónicos desaparecen.

En este mismo orden de ideas, Tyson y Jackson (2005), afirman que con la creación del consenso las dos partes llegan a un compromiso, concediendo puntos que consideran más favorables hasta que, finalmente, se llega a un acuerdo. En cierto sentido, esta etapa con frecuencia implica negociaciones intensas de las partes interesadas. El dilema del grupo se resuelve si uno de los bandos se convierte al punto de vista expresado por el otro. Esto se logra simplemente mediante el peso de la lógica, incluso las promesas.

Cuando el gerente educativo logra el consenso asegura la participación de todo el grupo en la decisión tomada, tal como lo demandan los diferentes roles que debe cumplir dentro de los centros educativos, que le permitan mantener unas buenas relaciones humanas.

\section{Metodología}

La metodología fue de tipo cuantitativa y enmarcada en la descripción desde la perspectiva que propuso evaluar las relaciones interpersonales entre docentes universitarios que ocupan cargos gerenciales en la convivencia escolar. Su acepción descriptiva responde a lo propuesto por Hernández y col (2014), quien afirma que describir una variable se hace tal como se presenta para ser evaluada. Así mismo siguió un diseño no experimental, transeccional y de campo. La población la constituyeron 115 docentes universitarios que ocupan cargos gerenciales en instituciones universitarias. Para la recolección de la información, se hizo uso de un instrumento tipo cuestionario y con 5 alternativas de repuestas (totalmente de acuerdo, de acuerdo, ni de acuerdo ni en desacuerdo, en desacuerdo y totalmente en desacuerdo)

\section{Resultados}

Posterior al análisis estadístico de los datos aportados por los investigados, los mismos se presentan en tablas con sus respectivos gráficos. 
Tabla 1. Análisis descriptivo de las relaciones interpersonales basadas en la participación

\begin{tabular}{|c|c|c|c|c|}
\hline ANÁLISIS/ CRITERIOS & $F R$ & $\%$ & $\overline{F R A}$ & $\% A$ \\
\hline Totalmente de acuerdo & 16 & 14 & 16 & 14 \\
\hline De acuerdo & 45 & 39 & 61 & 53 \\
\hline Ni de acuerdo ni en desacuerdo & 24 & 21 & 85 & 74 \\
\hline En desacuerdo & 20 & 19 & 105 & 93 \\
\hline Totalmente en desacuerdo & 10 & 7 & 115 & 100 \\
\hline MEDIA & \multicolumn{4}{|c|}{2,41} \\
\hline DESVIACION ESTANDAR & \multicolumn{4}{|c|}{$\mathbf{0 , 1 3}$} \\
\hline MEDIANA & \multicolumn{4}{|c|}{1,43} \\
\hline
\end{tabular}

Fuente: Elaboración propia (2019)

Gráfico 1: Análisis descriptivo de las relaciones interpersonales basadas en la participación

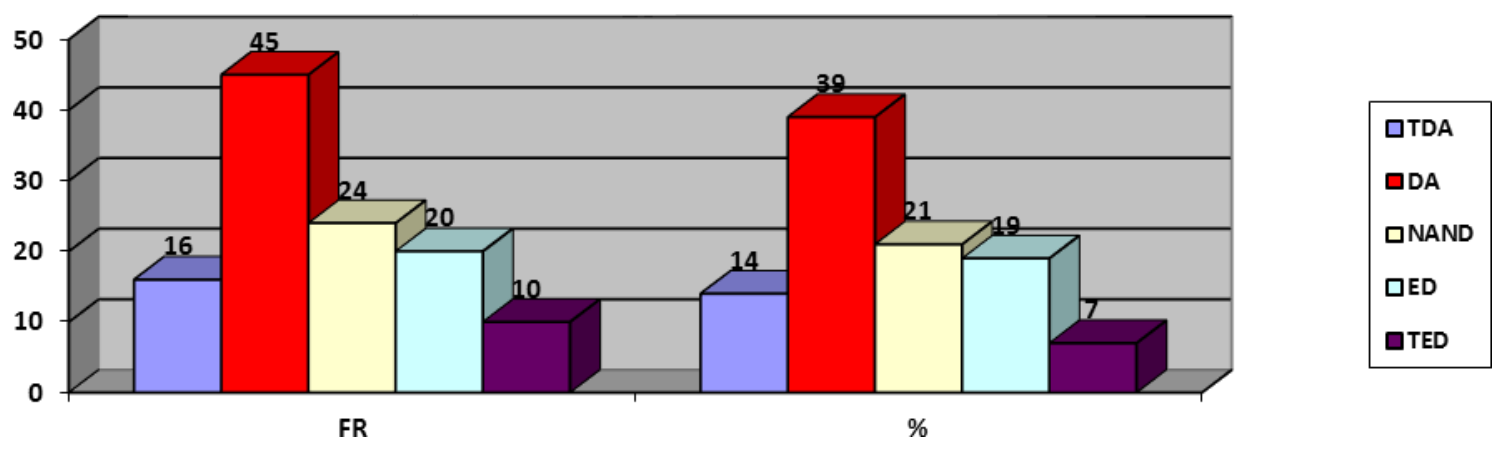

Fuente: Elaboración propia (2019)

En la tabla y gráfico 1, se presentan los resultados del análisis frecuencial y porcentual de las relaciones interpersonales basadas en la participación, en la que se observa que el 39\% del total 
de los docentes investigados coincidieron en opinar que estaban de acuerdo en que la gerencia educativa promueve la participación de los subordinados, toman en cuenta las opiniones de los subordinados cuando la situación así lo amerita, promueve la participación directa y unifica los criterios del que hacer educativo con sus subordinados. Seguida del $21 \%$ que señaló la alternativa ni de acuerdo ni en desacuerdo y el 19\% de los investigados que se inclinó por la alternativa en desacuerdo. Se destaca el hecho en la cual las alternativas extremas de totalmente de acuerdo y totalmente en desacuerdo alcanzaron los menores porcentajes de evaluación con el 14\% y $7 \%$ respectivamente.

Asimismo, se observa que el valor de la media fue de 2,41 con una desviación estándar de $\pm 0,13$, evaluándose como de alta presencia entre los investigados señalando que la misma puede variar entre las alternativas de acuerdo y en desacuerdo. La mediana con un valor 1,43 se ubicó por debajo de las medias señalando que existe una tendencia hacia las alternativas bajas de medición.

De acuerdo a estos resultados, se puede afirmar que en las instituciones de educación inicial s investigadas, aun cuando un alto porcentaje de los investigados señalan estar de acuerdo en que se debe promover la participación del personal, tomando en cuenta las opiniones de los mismos cuando la situación así lo amerita, promover la participación directa, ya que según Smith (2007), la participación es esencial en la relación directivo-empleado, además Koontz y Weihrich (2000), afirman que el tipo correcto de participación da por resultado al mismo tiempo motivación y conocimientos valiosos para el éxito de la empresa.

Tabla 2. Análisis descriptivo de las relaciones interpersonales basadas en la Resolución de Conflictos

\begin{tabular}{ccccc}
\hline ANÁLISIS/ CRITERIOS & FR & \% & FRA & \%A \\
\hline Totalmente de acuerdo & 3 & 2.5 & 3 & 2.5 \\
\hline De acuerdo & 35 & 30 & 38 & 32.5 \\
\hline Ni de acuerdo ni en desacuerdo & 57 & 49,5 & 95 & 82 \\
\hline En desacuerdo & 15 & 14 & 110 & 86 \\
\hline Totalmente en desacuerdo & 5 & 4 & 115 & 100
\end{tabular}




\begin{tabular}{|c|c|}
\hline MEDIA & 2,33 \\
\hline DESVIACION ESTANDAR & $\mathbf{0 , 1 2}$ \\
\hline MEDIANA & 2,31 \\
\hline
\end{tabular}

Fuente: Elaboración propia (2019)

Gráfico 2. Análisis descriptivo de las relaciones interpersonales basadas en la Resolución de Conflictos

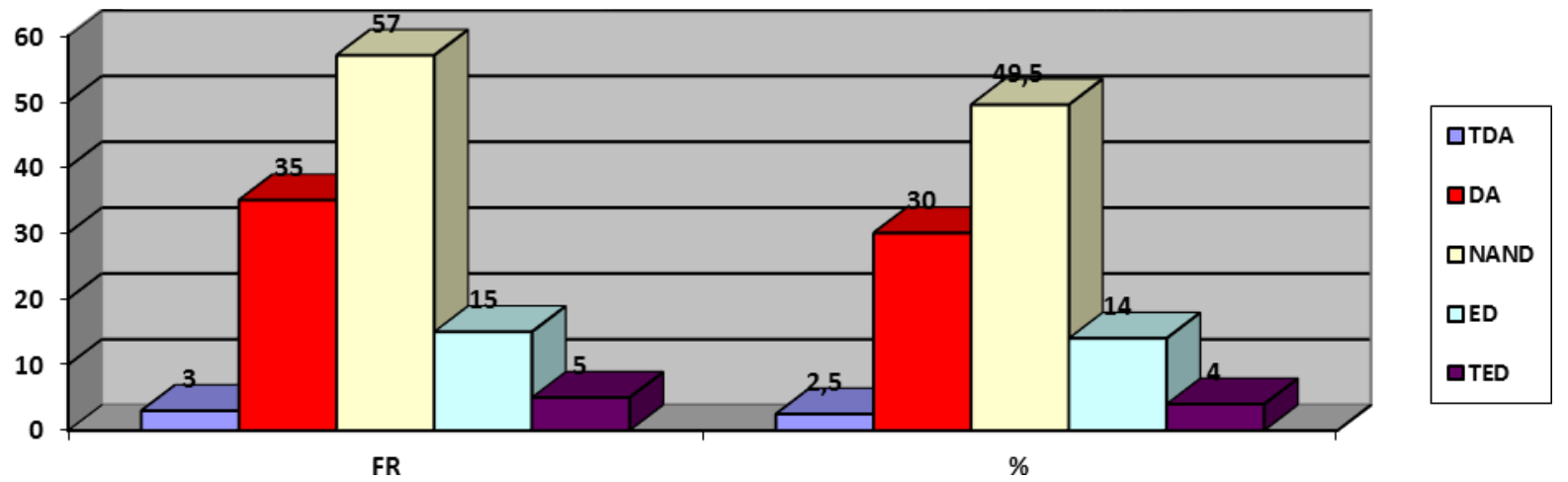

Fuente: Elaboración propia (2019)

En la tabla y gráfico 2, se presentan los resultados del análisis frecuencial y porcentual del indicador resolución de conflictos, en la que se indica que el 49,5\% del total de los docentes investigados coincidieron en señalar que estaban ni de acuerdo ni en desacuerdo en que frecuentemente la gerencia educativa combina las ideas para crear alternativas que permitan resolver problemas, ofrece soluciones creativas a los desacuerdos, promueve la integración de todo el personal a su cargo para resolver los conflictos y controla las diferencias personales que suceden entre los subordinados y el $30 \%$ de los directivos señaló la alternativa de acuerdo. Seguida del 14\% en opinión de los docentes que señaló la alternativa en desacuerdo. Se destaca el hecho en la cual las alternativas totalmente de acuerdo y totalmente en desacuerdo alcanzaron los menores porcentajes de evaluación con el 2,5\% y $4 \%$ respectivamente.

Asimismo, se observa que el valor de la media fue de 2,33 con una desviación estándar de $\pm 0,12$ evaluándose como de moderada presencia entre los investigados señalando que la misma puede variar entre las alternativas de acuerdo y en desacuerdo. La mediana con un valor 2,31 se ubicó 
ligeramente por debajo de las medias señalando que existe una tendencia hacia las alternativas bajas de medición.

Al respecto, Milkovich y Boudreau (2004), consideran que el conflicto debe dirigirse de manera que se protejan los derechos de los empleados y que los sistemas de resolución de conflictos deben ser más atractivos que las renuncias o demandas; en consecuencia de acuerdo a los resultados obtenidos en la investigación da la impresión que el gerente educativo no siempre es capaz de cambiar la situación que provoca los conflictos.

Tabla 3. Análisis descriptivo de las relaciones interpersonales basadas en la Creación del Consenso

\begin{tabular}{|c|c|c|c|c|}
\hline ANÁLISIS/CRITERIOS & $\overline{F R}$ & $\%$ & $\overline{F R A}$ & $\% A$ \\
\hline Totalmente de acuerdo & 9 & 10 & 9 & 10 \\
\hline De acuerdo & 48 & 40 & 57 & 50 \\
\hline Ni de acuerdo ni en desacuerdo & 33 & 27 & 90 & 67 \\
\hline En desacuerdo & 20 & 20 & 110 & 97 \\
\hline Totalmente en desacuerdo & 5 & 3 & 115 & 100 \\
\hline MEDIA & \multicolumn{4}{|c|}{1,82} \\
\hline DESVIACION ESTANDAR & \multicolumn{4}{|c|}{0,12} \\
\hline MEDIANA & \multicolumn{4}{|c|}{1,04} \\
\hline
\end{tabular}

Fuente: Elaboración propia (2019)

Grafico 3. Análisis descriptivo de las relaciones interpersonales basadas en la Creación del Consenso. 


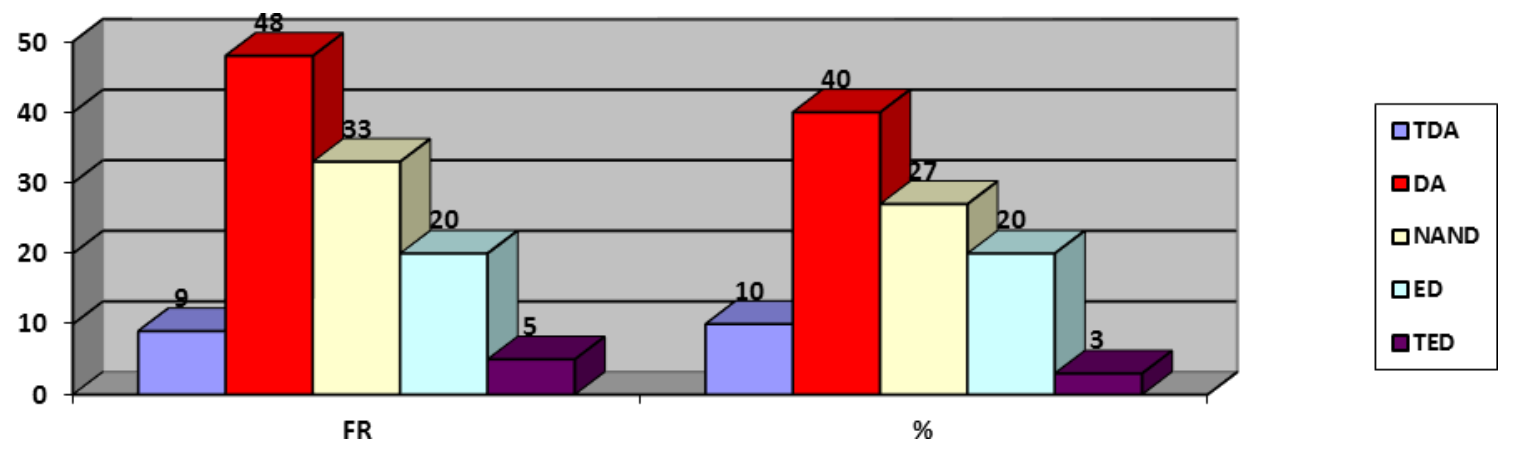

Fuente: Elaboración propia (2019)

En la tabla y gráfico 3, se presentan los resultados del análisis frecuencial y porcentual del indicador creación del consenso, en la que se indica que el $40 \%$ del total de los docentes investigados coincidieron estar de acuerdo en que la gerencia manifiesta actitudes para atenuar los conflictos, ayuda a las partes a sugerir nuevas ideas sobre la manera de poder resolver las situaciones conflictivas y escucha con interés a los subordinados para lograr el consenso, mientras que el $27 \%$ señalaron la alternativa ni de acuerdo ni en desacuerdo. Seguida del $20 \%$ que señaló la alternativa en desacuerdo y el $10 \%$ que indicaron la alternativa totalmente de acuerdo. El menor porcentaje se indicó para la alternativa totalmente en desacuerdo.

Así mismo, se observa que el valor de la media fue de 1,82 con una desviación estándar de $\pm 0,12$, evaluándose como de alta presencia entre los investigados señalando que la misma puede variar entre las alternativas ni de acuerdo ni en desacuerdo y en desacuerdo. La mediana con un valor 1,04 se ubicó por debajo de las medias señalando que existe una tendencia de opinión hacia las alternativas bajas de medición.

Al respecto, Willes (2006), expresa que cuando se busca el consenso es necesario interpretar las creencias de los demás para así poder determinar las áreas de acuerdos, sin embargo los resultados arrojados por la investigación no son muy satisfactorios, ya que evidencian que no siempre logran la creación del consenso y olvida o desconoce que el consenso sólo ocurre cuando todos los miembros del grupo se sienten realmente libres, y con la voluntad de expresar sus opiniones, puntos de vistas, sentimientos, desacuerdos, dudas, etc.; y cuando todos tengan de 
hecho el deseo de trabajar juntos, en el sentido de formar una solución-decisión que satisfaga realmente al deseo, intereses y motivaciones de todos. En este mismo orden de ideas, Tyson y Jackson (2005), afirman que con la creación del consenso se llega a un compromiso, concediendo puntos que consideran más favorables hasta que, finalmente se llega a un acuerdo.

\section{Conclusiones}

Existe acuerdo entre los docentes investigados, en que las relaciones interpersonales basadas en la participación deben ser consideradas para lograr los cambios y la orientación institucional, haciéndolo más receptivo, entendiendo que cuando se proyecta al grupo, genera mayor eficiencia y productividad, en tal sentido, cuanto más participa un miembro, más favorables son sus actitudes hacia el grupo y mayores son sus sentimientos de intereses y la identificación con el mismo.

La resolución de los conflictos en opinión de los investigados, señalaron estar ni de acuerdo ni en desacuerdo, en que el gerente lograba distinguir entre las diversas formas de conflicto a las diversas formas para tratar de resolverlos, generando pocas ideas para crear alternativas que permitan resolver problemas de relaciones interpersonales en la convivencia escolar.

En cuanto a las relaciones interpersonales de creación del consenso, se evidenció entre los docentes investigados que coincidieron en estar de acuerdo en que la gerencia manifiesta actitudes para atenuar los conflictos, ayuda a las partes a sugerir nuevas ideas sobre la manera de poder resolver las situaciones conflictivas y escucha con interés a los subordinados para lograr el consenso.

\section{Referencias}

1. Habermas, J. (2008). El futuro de la Naturaleza humana ¿Hacia una eugenesia liberal? Editorial Paidós. Barcelona.

2. Hernández R, Fernández C, Baptista P (2014). Metodología de la Investigación. Chile: McGraw - Hill. 
3. Ianni Gómez., L. (2017). MIRAMDA: Una propuesta educativa emergente desde la investigación. Revista Arbitrada Interdisciplinaria Koinonía, 2(3), 9-30. Recuperado de http://fundacionkoinonia.com.ve/ojs/index.php/revistakoinonia/article/view/49/36

4. Koontz, H. y Weihrich, H. (2000). Administración. Una Perspectiva Global. Décima Primera Edición. México: Editorial McGraw-Hill Interamericana S.A. de C.V.

5. Lemus, L. (2003). Administración, Dirección y supervisión. Editorial Kapelusz. Buenos Aires.

6. Lester, B. (2001). Curso de Management. Editorial Mc. Graw Hill. España.

7. Milkovich, G. y Boudreau, J. (2004). Dirección y administración de recursos humanos. Un enfoque de estrategia. Addison - Wesley Iberoamericana. México.

8. Smith, C. (2009). Guías para Supervisores. Editorial Trillas. México.

9. Tyson, S. y Jackson, T. (2005). La Esencia del Comportamiento Organizacional. Editorial Prentice Hall Hispanoamericana, S.A. México.

10. Wiemann, M. (2011). La comunicación en las relaciones interpersonales. Barcelona: UOC.

11. Wiles, K. (2006). Técnicas de Supervisión para mejorar Escuelas. Editorial Trillas. México.

\section{References}

1. Habermas, J. (2008). The future of human Nature Towards a liberal eugenics? Editorial Paidós. Barcelona.

2. Hernández R, Fernández C, Baptista P (2014). Investigation methodology. Chile: McGraw - Hill.

3. Ianni Gómez., L. (2017). MIRAMDA: An educational proposal emerging from research. Interdisciplinary Arbitrated Review Koinonía, 2 (3), 9-30. Recovered from http://fundacionkoinonia.com.ve/ojs/index.php/revistakoinonia/article/view/49/36 
4. Koontz, H. and Weihrich, H. (2000). Administration. A Global Perspective Tenth First Edition. Mexico: Editorial McGraw-Hill Interamericana S.A. from C.V.

5. Lemus, L. (2003). Administration, Direction and supervision. Kapelusz editorial. Buenos Aires.

6. Lester, B. (2001). Management Course Editorial Mc. Graw Hill Spain.

7. Milkovich, G. and Boudreau, J. (2004). Management and administration of human resources. A strategy approach. Addison - Wesley Iberoamericana. Mexico.

8. Smith, C. (2009). Guides for Supervisors. Editorial Trillas. Mexico.

9. Tyson, S. and Jackson, T. (2005). The Essence of Organizational Behavior. Editorial Prentice Hall Hispanoamericana, S.A. Mexico.

10. Wiemann, M. (2011). Communication in interpersonal relationships. Barcelona: UOC.

11. Wiles, K. (2006). Supervision techniques to improve schools. Editorial Trillas. Mexico. 